\title{
Thermal Metamaterials Make it Possible to Control the Flow of Heat at Will
}

\author{
Jiping Huang*
}

Accepted 11 December 2019

DOI: $10.30919 /$ esee 8 c 368

\section{Metamaterial physics might deserve a Nobel prize}

If the Nobel Prize can be used to measure whether a field is important or not, I would say that the field of metamaterial physics plays the same role as those fields that have been issued Nobel prizes in physics. See Fig. 1. Since the seminal article by V. G. Veselago (June 13, 1929September 15, 2018) in 1968 and especially the two other seminal articles by J. B. Pendry and coauthors in 1996 and 1999, the field of metamaterial physics has grown vigorously until today. With the aid of the new concept of metamaterials, many fundamental physics have been discovered in various branches of physics, ranging from optics/electromagnetics to elasticity/acoustics/mechanics/..... for wave systems, and from thermotics to particle dynamics for diffusion systems. As a result, various kinds of metamaterials were theoretically designed and experimentally fabricated in such branches. This volume focuses on the branch of thermotics, namely, thermal metamaterials. The phrase "thermal metamaterial" was first adopted in Ref. [1] to name thermal cloaks (shields) and relevant devices designed by using transformation thermotics for heat conduction (diffusion) studied in the five references. ${ }^{2-6}$ Owing to the existence of three ways of heat transfer (i.e., conduction, convection and radiation), nowadays the connotation of "thermal metamaterial" has naturally been extended to include metamaterials for controlling heat convection and/or radiation. Incidentally, the wording "thermal metamaterial" also contains some typical thermal metadevices (whose novel functions are realized mainly because of specific geometric structures), to comply with the common usage in the literature.

\section{Thermal metamaterial: past, present, and future}

In 2008, my group and Chen's group predicted the concept of novel thermal phenomena including thermal cloaking one after another., At the early stage (loosely speaking, before 2014) of thermal metamaterials, many experiments have been conducted to demonstrate the phenomenon of thermal cloaking under various conditions, see Refs. [59]. Accordingly, this field received plenty of popular attention ${ }^{10-12}$ (see also http://www.sciencemag.org/news/2012/05/heat-trickery-paves-waythermal-computers-).

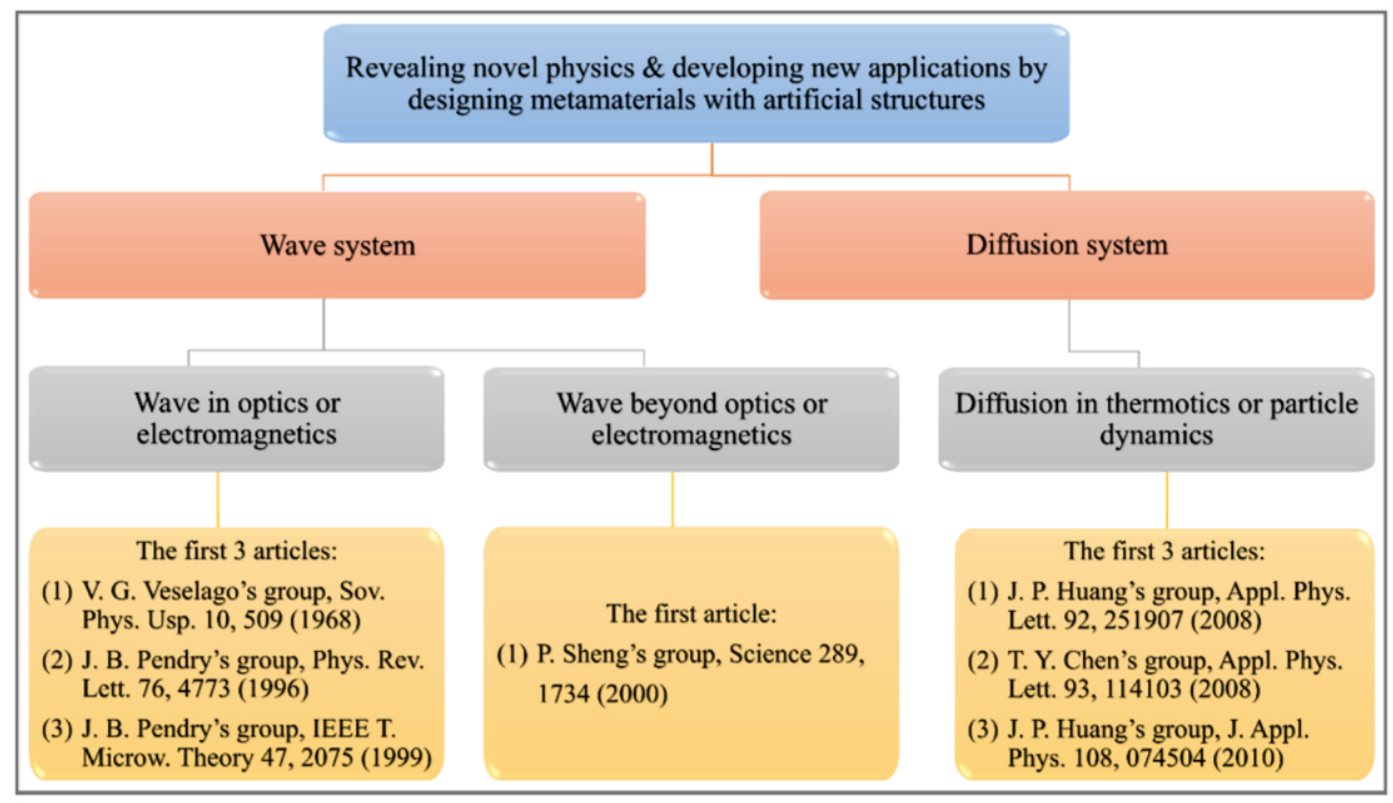

Fig. 1 A large number of novel physics and applications have arisen from metamaterials with artificial structures for wave systems and diffusion systems since 1968 and 2008, respectively. Both waves and diffusion are two important methods for transferring energy.

Department of Physics, State Key Laboratory of Surface Physics, and Key Laboratory of Micro and Nano Photonic Structures (MOE), Fudan University, Shanghai 200438, China

*E-mail: jphuang@fudan.edu.cn 
Thermal metamaterials mean those materials or devices with artificial structures that can be used to control heat conduction, convection and/or radiation in novel manners. In this case, geometric structure (rather than physical property) plays a dominating role. This fact makes thermal metamaterials different from other materials including thermoelectric materials, pyroelectric materials, magnetocaloric materials, and photo thermal conversion materials. For the latter, physical properties (rather than geometric structures) play dominating roles instead.

So far, thermal metamaterials have aroused enormous research interests, as also evidenced by Google search that shows the search of "thermal metamaterials" occupies $29.6 \%$ of all kinds of "metamaterials" as of August 13, 2019.

To celebrate the fruitful progress of thermal metamaterials and to prepare for the future challenges, I launched and chaired a National Conference on Thermodynamics and Thermal Metamaterials on July 18-19, 2019, in Fudan University, Shanghai, China. About 40 participants attended the first national conference, see Fig. 2. Fortunately for me, the editor-in-chief Prof. Bingyang Cao and his editorial team of ES Energy \& Environment greatly support the conference, and they also kindly accepted my proposal on publishing a special section about the topic "Thermal metamaterials or metadevices". As a result, we have received 17 review or research articles, which come not only from the attendees of the conference, but also from the researchers abroad. In this volume, we first publish 8 research articles and 1 review article. These articles cover the active areas of thermal metamaterials or metadevices, such as thermal cloaking, radiative cooling, programmable metamaterials, thermal rectification, thermal illusion, and thermal transparency.

\section{Useful theoretical physics and useful theoretical thermotics}

To design thermal metamaterials in the literature, analytic theories have been extensively developed with a special focus on transformation thermotics. Here I would prefer to call the transformation thermotics and its extended theories together as "theoretical thermotics", with an attempt to contribute them to the discipline of "theoretical physics (statistical physics)". This name could also remind the colleagues and latecomers to figure out the microscopic mechanisms for "theoretical thermotics" (that, after all, mainly describes macroscopic thermal theories for the time being), rather than to satisfy with the existing macroscopic theories.

In a word, theoretical thermotics describes the theory of transformation thermotics and its extended theories for the active control of macroscopic thermal properties of artificial systems, namely, metamaterials with artificial structures. Thus, theoretical thermotics is in sharp contrast to classical thermodynamics, which mainly comprises the four thermodynamic laws with a particular emphasis on the passive description of macroscopic thermal properties of natural systems.

Clearly, theoretical thermotics can help to arbitrarily design thermal metamaterials, which are further useful for engineering techniques and applications, ${ }^{13}$ say, for designing standard printed circuit boards, ${ }^{14,15}$ daytime radiative cooling, ${ }^{16}$ and so on.

Consequently, thermal metamaterials make it possible to control the flow of heat at will.

Notes: This editorial is adapted from the preface of my recent monograph. ${ }^{17}$

\section{References}

1. M. Maldovan, Nature, 2013, 503, 209-217.

2. C. Z. Fan, Y. Gao and J. P. Huang, Appl. Phys. Lett., 2008, 92, 251907.

3. T. Y. Chen, C. N. Weng and J. S. Chen, Appl. Phys. Lett., 2008, 93, 114103.

4. S. Guenneau, C. Amra and D. Veynante, Opt. Express, 2012, 20, 8207-8218.

5. S. Narayana and Y. Sato, Phys. Rev. Lett., 2012, 108, 214303.

6. R. Schittny, M. Kadic, S. Guenneau and M. Wegener, Phys. Rev. Lett., 2013, 110, 195901

7. T. C. Han, X. Bai, D. L. Gao, J. T. L. Thong, B. W. Li and C. W. Qiu, Phys Rev. Lett., 2014, 112, 054302.

8. H. Y. Xu, X. H. Shi, F. Gao, H. D. Sun and B. L. Zhang, Phys. Rev. Lett., 2014, 112, 054301.

9. Y. G. Ma, Y. C. Liu, M. Raza, Y. D. Wang and S. L. He, Phys. Rev. Lett., 2014, 113, 205501.

10. U. Leonhardt, Nature, 2013, 498, 440-441.

11. M. Wegener, Science, 2013, 342, 939-940.

12. P. Ball, Nat. Mater., 2012, 11, 566-566.

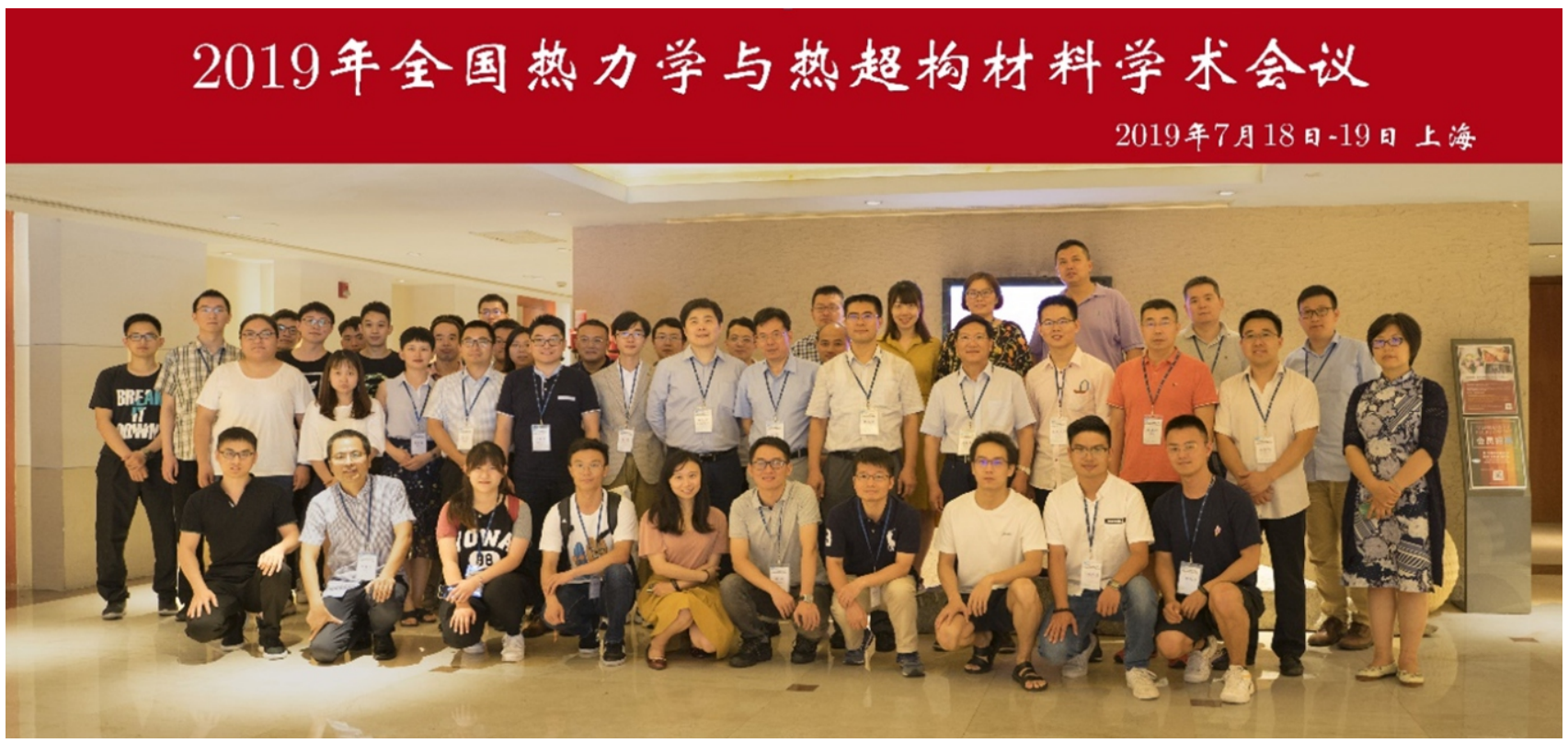

Fig. 2 Group photo: 2019 National Conference on Thermodynamics and Thermal Metamaterials, held on July 18-19, 2019, in Fudan University, Shanghai, China. 
13. J. P. Huang, Technologies for controlling thermal energy: design, simulation and experiment based on thermal metamaterial theories including transformation thermotics (in Chinese), Higher Education Press, Beijing, 2020 .

14. E. M. Dede, P. Schmalenberg, T. Nomura and M. Ishigaki, IEEE T. Compo. Pack. Man., 2015, 5, 1763-1774

15. E. M. Dede, F. Zhou, P. Schmalenberg and T. Nomura, J. Electron. Packag.,
2018, 140, 010904

16. Y. Zhai, Y. G. Ma, S. N. David, D. L. Zhao, R. N. Lou, G. Tan, R. G. Yang and X. B. Yin, Science, 2017, 355, 1062.

17. J. P. Huang, Theoretical thermotics: transformation thermotics and extended theories for thermal metamaterials, Springer, 2020.

Publisher's Note Engineered Science Publisher remains neutral with regard to jurisdictional claims in published maps and institutional affiliations. 